\title{
Desirability of a Teaching and Learning Tool for Thai Dance Body Motion
}

\author{
Worawat Choensawat ${ }^{1}$, Kingkarn Sookhanaphibarn ${ }^{1}$, Chommanad Kijkhun ${ }^{2}$, \\ and Kozaburo Hachimura ${ }^{3}$ \\ 1 School of Science and Technology, Bangkok University, Thailand \\ \{worawat.c,kingkarn.s\}@bu.ac.th \\ http://mit.science.bu.ac.th \\ 2 Suan Sunandha Rajabhat University, Thailand \\ 3 School of Science and Engineering, Ritsumeikan University, Japan \\ hachimura@media.ritsumei.ac.jp
}

\begin{abstract}
This paper investigates the desirability of using a teaching and learning tool for Thai dance in the context of higher education. Unlike the Western dances where dance notation have been widely used for recording the dance body movement, students in Thai dance classes have to memorize a series of body movements by observation from their teachers. In Thai dance communities, dance notation is very new, and few of professional people in Thai dance understand and use it to record the Thai dance body movement. In this paper, we demonstrate the adaption of a notation system to describe Thai dance and introduce a learning tool for facilitate students to understand the notation. Our presented tool for teaching and learning Thai dance is as a result from a collaboration research between researchers from performing arts and computer science. We measure the desirability of our tool with four Thai dance schools dispersedly located in the north and middle of Thailand, and we receive a promising feedback from them.
\end{abstract}

Keywords: Desirability Methodology, User Evaluation, Dance notation, Labanotation, LabanEditor, Multimedia Tool, Dance Animation.

\section{Introduction}

Thai dance, like many forms of traditional Asian dance, can be divided into two major categories that correspond roughly to the high art (royal dance) and low art (folk dance) distinction [9]. In this study, we are interested in the high art or performing art. One of famous high arts is called Khon, which is a traditional most sophisticated form of Thai masked drama which combines gracefulness with masculinity in its dancing and singing. Unlike tangible cultural assets like paints and archaeological site, dances are intangible cultural property 6. The challenge with intangible heritage is that it is not preserving an object, but a process to pass it on to the next generation. Textbooks for teaching Thai dance are literately written with the context of drawing and photography material

A. Marcus (Ed.): DUXU/HCII 2013, Part II, LNCS 8013, pp. 171-179, 2013.

(C) Springer-Verlag Berlin Heidelberg 2013 

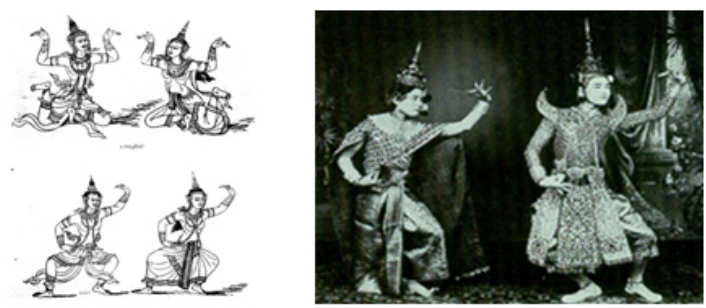

Fig. 1. Textbook used for Thai dance

to illustrate a movement of dancers as shown in Fig. 1. However, the rhythm cannot be expressed. Therefore, the knowledge of Thai dance has been taught to students by observation and imitation of body movements.

It is widely acceptable that Labanotation is a useful tool for human movement recording, choreography and dance training [7]. Labanotation is one of the most common movement notation systems. From unique characteristic of Thai dance, most people understand that it would be difficult to handle this kind of stylized traditional movement with Labanotation. Even though it is possible, a resulting notation would become very complicated.

Because of the complexity of Labanotation, it is not an easy task to introduce this new learning method based on the notation scheme to the dance community in Thailand. To overcome the barrier, one of the solution is to use a computeraided tool to help new learner in understanding the Labanotation. We have been developing a system for preparing Labanotation scores and displaying 3D animation associated with the score 234 . In this paper, we measure the desirability of the tool for describing and reproducing Thai dance. The survey experiment was conducted on over 200 students and 17 teachers in four well-known schools in Thai dances.

\section{Teaching and Learning Tools in Dance Communities}

For anyone who can read music, reading a musical score is much more straightforward than trying to pick out the sounds of all the instruments by ear, and to figure out the composer's intention from an audio recording alone. The same is true for dance notation. Dance notation captures the choreographer's creative idea, just as music notation captures the composer's creative idea. Dance notation can be thought analogous to presenting dance that is an output of what the choreographer wants and what the dancer actually does. Several dance notations were devised and have been used for recording and educating dance. The most commonly adopted notation systems are Labanotation [7] and Benesh Notation [1]. Among them Labanotation is most popular. 


\subsection{Labanotation}

Labanotation is the system of recording human movement devised in the 1920's. Labanotation scores are similar to musical scores that record the human body movement by using graphical symbols. Labanotation score is drawn in a form of vertical staffs where each column represents the motion of a body part as shown in Fig. 2 (a). The symbols are placed on the vertical staff; the horizontal dimension of the staff represents the parts of the body, and the vertical dimension time. The center line of the staff represents the center line of the body; symbols on the right represent the right side of the body, symbols on the left, and the left side. Fig. 2 (b) shows the basic arrangement of columns in the staff.

The direction of movements are identified by a set of direction signs as shown in Fig. 3. The shape of symbol represents the direction of horizontal motion. A shade of direction sign shows the level of a movement. The motion of each body part is expressed by a sequence of symbols placed in the corresponding column.

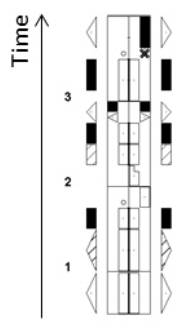

(a)

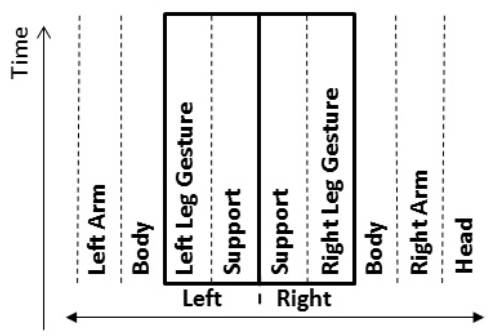

(b)

Fig. 2. Labanotation score: (a) Example of Labanotation score, (b) Definition of staffs in each column

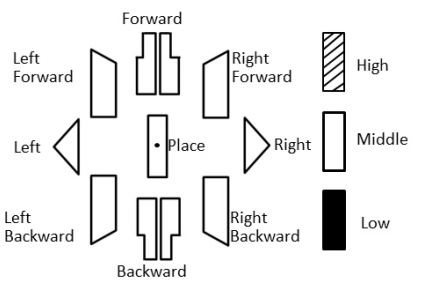

Fig. 3. Symbols for direction signs 


\subsection{LabanEditor}

LabanEditor was introduced by Hachimura and his research team 28 as an interactive graphical editor for writing and editing Labanotation scores. By using LabanEditor, a user can input/edit dance movement and replay the animation of human body motion corresponding to the Labanotation score via 3D computer graphics. There are also other software, for example LabanWriter [5] is the most widely used Labanotation editor. LabanWriter is only for preparing Labanotation scores and recording them in digital form. It does not provide a function for displaying character animations corresponding to the notation. LabanDancer (developed by Wilke et al. [10]) is a LabanWriter scores to 3D animation translation tool, LabanDancer does not have function for preparing Labanotation scores.

At present, LabanEditor can serve the needs of dance community because its full feature of description and reproduction. Fig. 4 shows a user interface of LabanEditor. While displaying the CG animation of the score, we can observe the animation as well as the red horizontal line cursor, moving upward as the

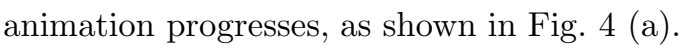

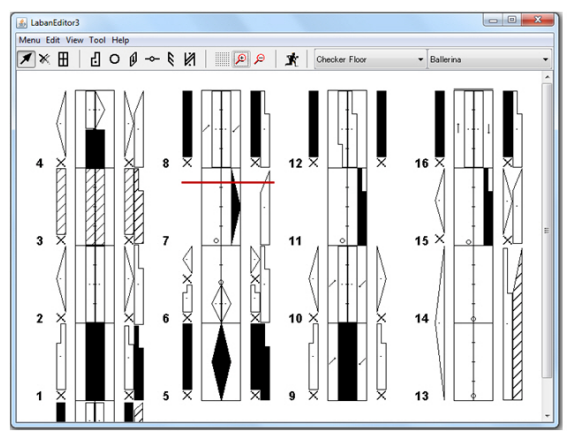

(a)

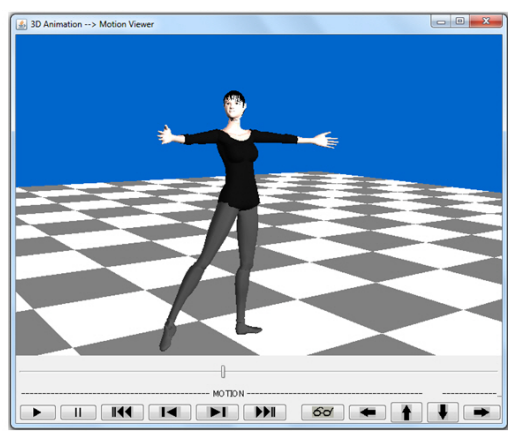

(b)

Fig. 4. User interface of LabanEditor: (a) Main editing window of LabanEditor, (b) CG animation display window

\section{Thai Dance Notation System}

Labanotation is able to describe very detail of movement such as a finger movement; however, the combination of symbols becomes too complex to understand. It would be very difficult for introducing this kind of complex language to Thai dance communities. To solve the problem, we develop a set of new symbols that can describe all movements of the fundamental Thai dance. As a rule of thumb, we have the design decision in terms of efficiency, comprehensibility, and advantage of this new notation system. It is called Thai dance notation system. For example, such a hand pose in Fig. 5 (a) can be written with our symbols as 


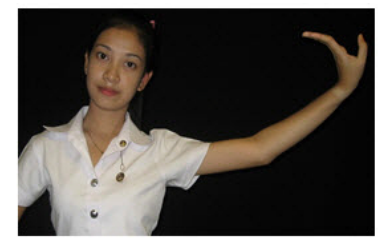

(a)

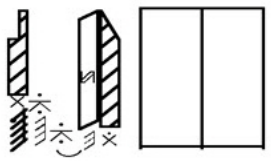

(b)

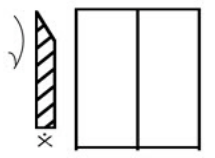

(c)

Fig. 5. Thai notation for hand gesture (Wong): (a) Hand gesture, (b) Standard Labanotation, and (c) Thai notation

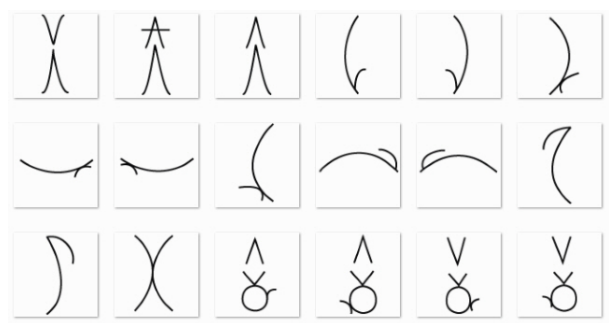

Fig. 6. New symbols for describing Thai dance

shown in Fig. 5 (c). For the Thai dance notation system, most of new symbols (Fig. 6) can represent all basic hand gesture, and the others try to describe a detail of foot movement that are very special and important of Thai dance (Fig. 7). Essentially, these symbols can picture the specific posture of classical Thai dance, such Jiib, Wong and foot lifting. To make the Thai dance notation system compatible with standard Labanotation, we also write the definition of all new symbols with a group of standard symbol of Labanotation.

\section{Measuring Desirability}

\subsection{Participants}

In context of higher education, we selected four Thai dance schools that are well-known in the north and middle of Thailand. The amount of students and teachers involving in our study is 202 and 17, respectively. All of participants have already had a basic skill of Thai dance, but none of them know Labanotation before. This is because most of Thai dance schools do not have a class of Western dances, where students will learn Labanotation.

\subsection{Tools}

The questionnaire has three parts: personal information, selecting words, and writing comments/ suggestions. There are totally Thai 24 words for both positive and 

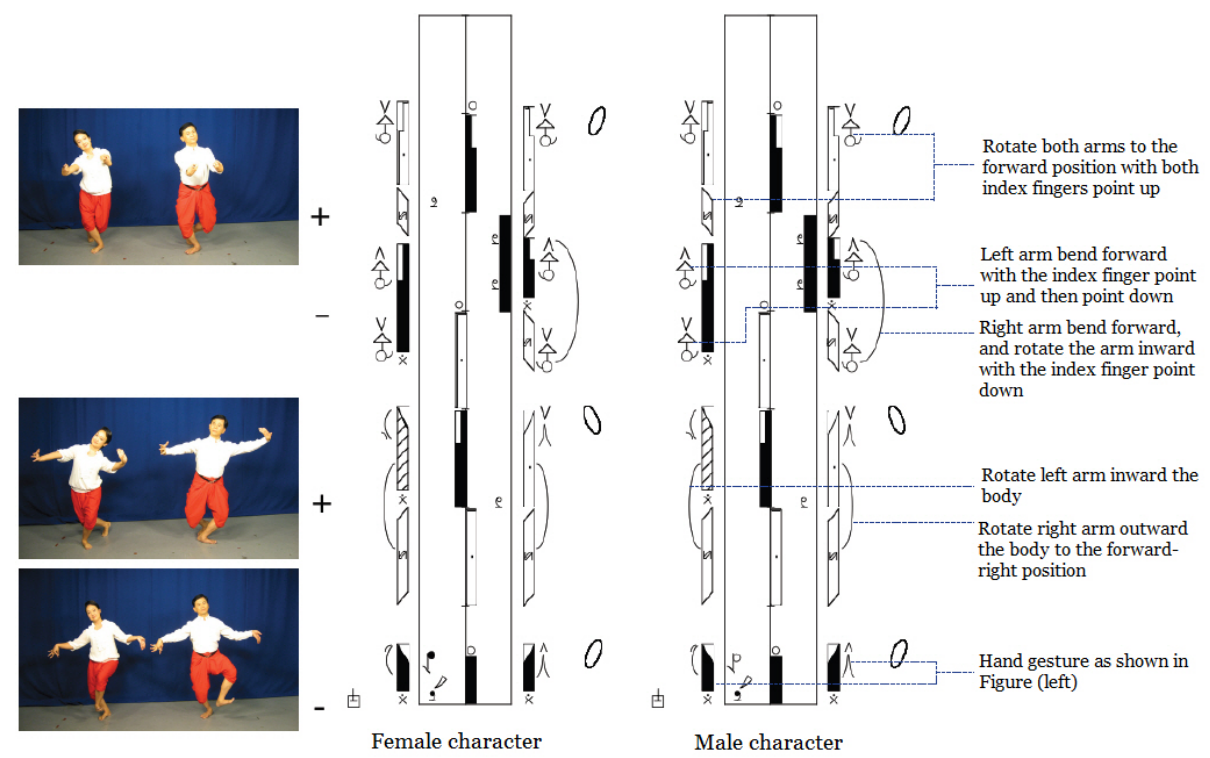

Fig. 7. Example of scores produced from Thai dance notation system

negative words in fairly balance (Translation into English is shown in Table. 1). For selecting words, subjects have to choose any words (subjects can choose more than one) that can explain the software.

Table 1. Choose word(s) for suitably describing our tool

$\square$ appealing
$\square$ attractive
$\square$ boring
$\square$ common
$\square$ complex
$\square$ confusing

$\square$ creative
$\square$ desirable
$\square$ difficult to use
$\square$ easy to use
$\square$ effective
$\square$ frustrating

$\square$ fun

$\square$ high quality

$\square$ inefficient

$\square$ low quality

$\square$ not valuable

$\square$ time-consuming

\subsection{Procedure}

For each school, we gave them a tutorial and demonstration of the teaching and learning tool for Thai dance. The procedure was divided into three sessions: i) tutorial of Labanotation, ii) introducing Thai dance notation system, iii) demonstrating LabanEditor with examples of ballet and Noh. Total time of a whole procedure was approximately 3 hours. After presentation, we conducted the survey experiment by using a questionnaire that subjects had about a few minute 
for this task. In addition, subjects were allowed to write any feedback about the teaching and learning tool for Thai dance.

The first and second sessions were conducted by Kijkhun who is a professional in Thai dance and a specialist of Labanotation teaching. The aim of the first session was to give the subjects an impression how the notation looks like and how to read the basic notation score. All participants had to practice with the fundamental symbols by reading the score and trying to move their body. In the second session, we demonstrated how to utilize the Labanotation for Thai dance and introduced Thai notation system to the subjects. For the last session, we demonstrated a computer-aided tool to accelerate them about memorizing all notation, and we also presented a case study of Ballet and Noh dance.

\section{Results and Discussion}

Fig. 8 shows the desirability of the software for describing and reproducing Thai dance body motion. Since Thai dance has not been described in a unified approach. For standardization, we have investigated to adopt the Labanotation for describing Thai dance. Since the notation has been never used for Thai dance, the understanding the notation is very difficult, and it will be a barrier for learning Thai dance. One solution is to investigate a computer-aided tool such as LabanEditor. LabanEditor is an interactive system for inputting/ editing Labanotation scores and displaying 3D CG character animation associated with the scores.

In this paper, we measure the desirability of this tool for describing and reproducing Thai dance. The survey experiment was conducted on over 200 students and teachers in four well-known schools in Thai dances. The results show that about $70 \%$ of subjects think that the software has usability, desirability, creativity, and fun. Feedbacks from subjects who are teachers in Thai dance shows more desirable to use the tool than those from students, and less than $5 \%$ of the subjects are unwilling to use the tool.

From the results, the barrier of using the technology is slightly found with the chosen words like frastrating, unconventional, confusing. However, the percentage is about $12-15 \%$ that is under expectation from our experience in art and humanity area. Moreover, there are some subjects who selected two conflicting words such as (too technical and easy to use), (complex and time-saving). The reasons are about the subject give their opinion on both Labanotation and the software that the notation is complex and too technical, but the software is easy to use and time-saving.

In the future work, we will create a content that includes both the score in Thai dance notation system and the corresponding animation. After completing the content, we will promote our tools in Thai dance schools and conduct the user evaluation. 


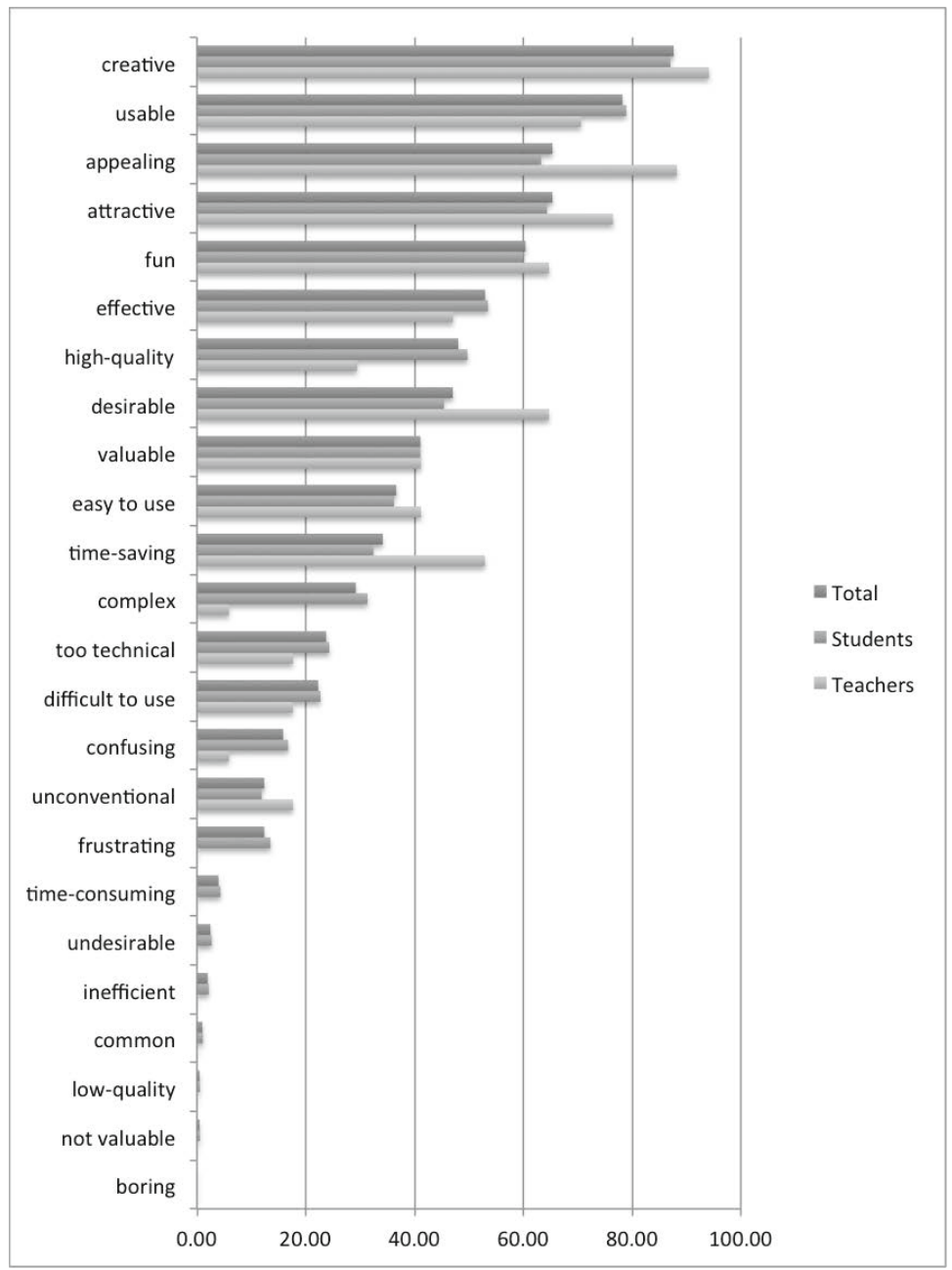

Fig. 8. Chart of chosen words which can explain the tool where number of teachers and students is 17 and 202 , respectively

\section{References}

1. Benesh, R., Benesh, J.: Reading Dance: The Birth of Choreology. McGraw-Hill Book (1983)

2. Choensawat, W., Takahashi, S., Nakamura, M., Choi, W., Hachimura, K.: Description and reproduction of stylized traditional dance body motion by using labanotation. Transactions of the Virtual Reality Society of Japan 15(3), 379-388 (2010) 
3. Choensawat, W., Takahashi, S., Nakamura, M., Hachimura, K.: The use of labanotation for choreographing a noh-play. In: 2011 Second International Conference on Culture and Computing (Culture Computing), pp. 167-168. IEEE (2011)

4. Choensawat, W., Takahashi, S., Nakamura, M., Hachimura, K.: Labanohtation: Laban meets noh. In: ACM SIGGRAPH 2012 Posters, p. 5. ACM (2012)

5. Fox, I., Ryman, R., Calvert, T.: Documenting dance for the 21st century: A translation interface between labanwriter and life forms. In: Twenty-Second Biennial Conference of the International Council of Kinetography Laban, July 26-August 2, vol. 26, pp. 193-205 (2001)

6. Hachimura, K.: Digital archiving of dancing. Review of the National Center for Digitization (Online Journal) 8, 51-66 (2006)

7. Hutchinson, A.: Labanotation. Dance Books (1996)

8. Kojima, K., Hachimura, K., Nakamura, M.: Labaneditor: Graphical editor for dance notation. In: Proceedings of the 11th IEEE International Workshop on Robot and Human Interactive Communication, pp. 59-64. IEEE (2002)

9. Rutnin, M.: Dance, Drama, and Theatre in Thailand: The Process of Development and Modernization. Silkworm Books (1996)

10. Wilke, L., Calvert, T., Ryman, R., Fox, I.: From dance notation to human animation: The labandancer project. Computer Animation and Virtual Worlds 16(3-4), 201-211 (2005) 